\title{
The Impact of a Medical Toxicology Fellowship on the Training of Future Emergency Medicine Residents
}

\author{
David H. Jang
}

Published online: 5 October 2010

(C) American College of Medical Toxicology 2010

What is the impact of a medical toxicology fellowship on the training of future emergency medicine residents? While I do realize that not all fellows come from an emergency medicine background, many medical toxicology fellowships are closely associated with an emergency medicine residency (also our boards fall under the American Board of Emergency Medicine as well as two other certifying organizations) [1]. No matter where the fellowship, a medical toxicology fellowship is very complicated. Aside from clinical duties including bedside consults to taking call, we also participate in research, learn to write, and also read copious amounts of journals to stay abreast of all the current literature $[2,3]$. As I am now starting my second-year fellowship, I look back at all the times I have taken call and reminisce on some of the cases I have had. I will highlight two contrasting cases I had one night which reminds me of another important, but sometimes forgotten function of fellowship - education.

The first case is one that stands out in my mind as a reminder of the importance of our role in the education of other health care providers. I remember receiving a call one night from a small local hospital that had a patient present

Editor's note: David H. Jang is a senior fellow-in-training (FIT) editorial board member at the New York City Poison Center and New York University. The opinions stated in this section are those of the author and do not necessarily reflect the opinions of the Journal of Medical Toxicology (JMT) or the American College of Medical Toxicology (ACMT).

\section{H. Jang $(\bowtie)$}

New York University,

New York, NY, USA

e-mail: Jangd01@nyumc.org

D. H. Jang

New York City Poison Center,

New York, NY, USA with a benzodiazepine overdose. The patient was a young woman who got into a fight with her boyfriend and, in a rash gesture, overdosed on her lorazepam. She presented with "coma with normal vital signs". The attending physician administered his version of the "coma cocktail" with $2 \mathrm{mg}$ of naloxone followed with $2 \mathrm{mg}$ of flumazenil. Soon after being administered flumazenil, the patient developed seizures refractory to benzodiazepines, requiring endotracheal intubation and the use of propofol. It was at this point that I received the call from the ED attending for guidance. Needless to say, the damage was done. The patient had a prolonged hospital course secondary to the complications of intubation and eventually was discharged. When I asked this particular health care provider at that time what was his rationale (in a non-judgmental tone of course) for administering both naloxone and flumazenil, his response was, "That was the way I was taught".

Later in the night, I received a call from a recent graduate of the same emergency medicine residency where I am doing my fellowship about a similar case involving a young patient who also overdosed on a sedative-hypnotic. This patient also had a classic benzodiazepine picture of "coma with normal vital signs". This recent graduate called early in the patient's course and, needless to say, I was on edge from the other case I mentioned above. He presented the case and described his management which consisted of supportive care. He stated that he did not feel strongly about using flumazenil. He reasoned that the benefit would not outweigh the risk of potentially inducing a withdrawal seizure in someone he felt was chronically on a benzodiazepine. I asked the recent graduate where he learned about the proper use of flumazenil. He mentioned that he remembered about the use of flumazenil from a lecture one of our fellows gave at grand rounds during his residency. 
It did not strike me until later how two health care providers could choose two contrasting management styles (one probably detrimental to the patient) based on the education they received during training. There are also other examples of cases which illustrate the importance of education with regard to training, in particular, when it comes to management of the poisoned patient. While the field of emergency medicine is broad and it encompasses many other fields, management of the poisoned patient is an important aspect. I was interested to see if there was any literature related to this topic and, despite an extensive search, could not locate much in regard to resident education related to a medical toxicology fellowship.

Despite the lack of "evidence" based on my own experience over the last year, I do believe that as medical toxicology fellows, we have an impact on other health care providers in the management of the poisoned patient. At the fellowship where I am currently training, we have an opportunity to lecture to various health care providers including pharmacists, residents from various fields such as pediatrics and emergency medicine, as well as to the lay public. Based on my experience, imparting practical knowledge to health care providers from various backgrounds is an art form. I do admit I sometimes forget the importance of teaching when I am working in a busy emergency department shift where I am trying to "move the meat". Sometimes, all it takes is an eager medical student who presents a patient that is clearly anticholinergic. That student may be completely fascinated with something we may take for granted.

\section{References}

1. Wax PM, Donovan JW (2000) Fellowship training in medical toxicology: characteristics, perceptions, and career impact. Clin Toxicol 38:637-642

2. Wax P, Ford M, Bond G et al (2004) The core content of medical toxicology. Ann Emerg Med 43:1-6

3. Schier JG, Rubin C, Schwartz MD (2010) Public health partnerships in medical toxicology education and practice. Am J Prev Med 38(6):667-674 